P\&A Año 2, N.2

enero-junio de 2017

pp. $77-86$

\title{
Resumen
}

El estudiante de Arquitectura afronta hoy la ausencia de consenso académico en el diseño. A menudo, trabaja con nociones vagas y desconoce el significado preciso de conceptos importantes, como lo son forma y figura. La crisis en la teoría formalista del arte ha desligado a la disciplina de su carácter visual. El lugar de la mirada lo ocupan contenidos semánticos, basados en conceptos, que se ubican en los márgenes de la disciplina. La figuratividad que se desprende de la expresión personal entra en conflicto con el orden que caracteriza al arte de construir. Por ello, se propone crear conciencia estética a través de la reconstrucción de obras ejemplares. En el presente texto, esta evaluación se contrasta con la práctica académica en Perú y se exploran posibles mejoras pedagógicas para el Taller Básico 2 de diseño arquitectónico de la Universidad Ricardo Palma, en Lima.

Palabras clave: Forma, figura, creatividad, innovación, enseñanza del diseño

\section{Distinción entre forma y figura: Su aplicación a la enseñanza en el Taller Básico 2 de Diseño Arquitectónico de la Universidad Ricardo Palma*}

\author{
Distinction between form and figure: Its application in teaching during the Second \\ Basic Workshop of Architectural Design of Ricardo Palma University \\ Arq. Óscar García Pérez**
}

Recibido: 10 de abril de 2017

Aceptado: 5 de junio de 2017

\begin{abstract}
The Architecture student currently faces the absence of academic consensus in regards to design. Usually, he works with vague notions and ignores the precise meaning of important concepts, which form and figure are. The crisis of the formalist theory of art has disconnected architecture from its visual character. The position of the gaze is now occupied by semantic contents, based on concepts, which are located in the margins of the discipline. What is figurative and comes from personal expression conflicts with the order that characterizes the art of building. Therefore, the creation of aesthetic awareness is proposed through the reconstruction of exemplary works. In this text, such an evaluation is contrasted with the academic practice in Peru and possible pedagogical improvements are explored in regards to the Second Basic Workshop of Architectural Design of Ricardo Palma University, in Lima.
\end{abstract}

Keywords: Form, figure, creativity, innovation, design teaching

\footnotetext{
* Este texto ha sido elaborado sobre la base del material de avance del tema de investigación con que se inicia la tesis doctoral del autor, dentro de la Escuela Técnica Superior de Arquitectura de Madrid (ETSAM). Es una investigación autofinanciada. Se declara no tener conflicto de intereses en la publicación de este artículo.

** Arquitecto por la Universidad Politécnica de Madrid (UPM). Docente en la Facultad de Arquitectura y Urbanismo de la Universidad Ricardo Palma (URP) en Lima, donde es jefe del Taller Básico 2 de Diseño Arquitectónico.
} 


\section{El mundo contemporáneo[...]abandona las formas exteriores en su expresión escue- tamente epidérmica y decorativa para tomarlas como productos de un fondo en comunicación con la sustancia. \\ Agrupación Espacio Expresión de Principios}

\section{Aprender a mirar}

Los estudiantes de Diseño enfrentan numerosas dificultades en su proceso de aprendizaje. La mayoría son problemas globales que afectan, en el ámbito de la enseñanza superior, a universidades en todo el mundo, con independencia del prestigio adquirido en otras ramas del saber. Algunas dificultades tienen su origen en los postulados posmodernos, los cuales han causado gran confusión en torno a las ideas centrales de la arquitectura.

La desconexión que en los últimos tiempos atraviesa la disciplina de su matriz artística, ha desviado su atención hacia soluciones conceptuales de escaso recorrido estético. La enseñanza del diseño ha olvidado nociones básicas de la teoría del arte. Por ello, si se desea delimitar el problema y proponer soluciones, habrá que acotar los términos difusos y definirlos con precisión.

Con el paso del tiempo, se ha confundido la idea de forma (noción estructurante) con la de figura (noción significante). Esto es decisivo, porque -como señala Alan Colquhoun (2000) - la famosa disyuntiva desplegada en torno a la forma y la función ya no se ubica en el centro del debate de la arquitectura contemporánea: "La dialéctica fundamental no parece ser más aquella entre forma y función, sino entre forma y otra entidad, a la cual llamaré figura" (p. 90). Además, la mayor parte de estudiosos de la arquitectura han empleado estos términos de manera indeterminada, con el consiguiente equívoco por parte de sus lectores. Es frecuente nombrar como formalismo a los excesos figurativos del diseño. Así, se ha convertido en un término peyorativo, cuando en realidad la forma es un concepto trascendental para la generación artística y arquitectónica.
Aunque la arquitectura moderna se asienta en la idea de forma, ha triunfado su interpretación funcional. Sin embargo, el formalismo puede volver a arrojar luz sobre algunas interrogantes que ocupan un papel central en la concepción de la arquitectura en el presente. Por ello, esta teoría sirve de referencia intelectual para el Taller Básico 2 de Diseño Arquitectónico en la Facultad de Arquitectura y Urbanismo de la Universidad Ricardo Palma (URP), en Lima. Este taller, ahora en proceso de renovación, plantea dos objetivos principales: aprender a mirar, estimulando al estudiante para que adquiera criterio de forma; y evidenciar que la enseñanza no procede tanto de la información que transmiten los docentes como del entendimiento del valor de la tradición actualizada del arte de construir.

\section{Las nociones de forma y de figura}

En estos momentos, asistimos a un malentendido en arquitectura: pensar que se entiende bien lo que se dice cuando se habla de forma. Aunque su comprensión es crucial para la enseñanza y el aprendizaje del proyecto, el término se confunde en castellano con figura. Podría decirse que son sinónimos. El significado ordinario de la palabra forma es de alcance universal para todos los hablantes, pero no lo es el discernimiento de esta idea cuando se inserta en la teoría del arte. A este respecto, Helio Piñón (2006) señala lo siguiente: "Considero esencial detenerme un momento en el concepto de forma: una noción que se usa constantemente y casi siempre en su acepción vulgar -aquella que la aproxima a la idea de figura- llegando incluso a identificarse con ella" (p. 38).

El Diccionario de la Lengua. Española define forma como la "configuración externa de algo", mientras que figura es la "forma exterior de alguien o de algo" (2014, párr. 1). Son definiciones cruzadas donde un concepto apunta al otro: la forma es una configuración y la figura es una forma. No es de extrañar entonces que una persona ajena al mundo del arte las use de modo indistinto, asumiendo que son equivalentes. El problema estriba en 
que la confusión también afecta a los profesionales del Diseño.

Es importante subrayar que el término latino forma es muy antiguo y que aparece sin apenas modificaciones en varias lenguas indoeuropeas. Wladislaw Tatarkiewicz destaca en Historia de Seis Ideas (2015) la existencia de al menos cinco significados diferentes para la noción de forma, desde Platón hasta nuestros días, que son o han sido operativos en el ámbito de la estética. Estos significados resumidos son los siguientes:

-Forma A: Es la disposición de las partes. Es una noción abstracta. Su opuesto son los elementos.

-Forma B: Es lo que se da directamente a los sentidos y atiende a una experiencia concreta. Su opuesto es el contenido.

-Forma C: Es el contorno de un objeto y equivale a la noción común de forma o de figura (primera acepción de los términos, según la RAE). Su opuesto es la materia.

-Forma D: Es la esencia conceptual de un objeto (Aristóteles). Su opuesto son sus rasgos accidentales.

-Forma E: Es la forma a priori, la contribución de la mente a la percepción del objeto (Kant). Su opuesto es lo que se le da a la mente desde fuera a través de la experiencia.

Los tres primeros significados proceden directamente de la teoría del arte, mientras que los dos últimos se incorporan a la estética a partir del pensamiento filosófico.

Pero si la forma ha ocupado siempre un papel central en teoría del arte -trascendiendo la acepción que adquiere en el lenguaje cotidia-

1 Carlos Martí (2000) expone la diferencia que existe, en arquitectura, entre los términos forma y figura: "La clave de esta distinción parece estar ya implícitamente contenida en la doble acepción que posee la palabra forma, en el ámbito arquitectónico, según se la considere como transcripción del término griego eidós o bien del alemán Gestalt. En el primer caso, la forma se identifica con la esencial constitución interna de un objeto, y alude a la disposición y ordenación general de sus partes, de manera que la forma se identifica con el moderno concepto de estructura; en el segundo caso, la forma se refiere a la apariencia del objeto, a su aspecto o conformación externa, de modo que se convierte en sinónimo de figura" (p. 8). no-, la figura ha recibido históricamente un menor examen. No obstante, los significados de figura (entre los que se incluye el correspondiente a la forma $\mathrm{C}$ ) difieren por completo de la noción kantiana de forma o forma E, aquella que es susceptible de integrar en su seno el orden complejo de la arquitectura.

\section{La crisis del formalismo y de la arquitectura moderna}

Fue la forma kantiana o forma $\mathrm{E}$ la que dio origen a una corriente conocida en teoría del arte como formalismo o pura visualidad (Sichtbarkeit), que tuvo gran popularidad en la Alemania del siglo XIX, y causó un fuerte impacto en la aparición de las vanguardias artísticas centrípetas ${ }^{2}$ y de la arquitectura moderna.

La estética formalista fue enunciada en primera instancia por Immanuel Kant (2012) en su Crítica del Discernimiento ${ }^{3}$ de 1790 , pero hubo que esperar hasta finales del siglo XIX para que Konrad Fiedler inaugurase esta corriente de pensamiento en el ámbito de la historia del arte. La tesis principal del autor estriba en considerar el arte como un problema de conocimiento, que equivale -siguiendo a Kant- a una toma de conciencia de las sensaciones percibidas que la mente conforma a través de la subjetividad. El arte ya no se limita a reproducir la belleza de la realidad externa, sino que él mismo constituye una nueva realidad. De esta idea deriva la tesis que propugna que es la forma la que otorga identidad a las artes plásticas. De Fusco (1976) lo enuncia de la siguiente manera: "Esta forma que es también, al mismo tiempo, contenido, no debe expresar más que a sí misma; el resto de lo que expresa, en su calidad de lenguaje ilustrativo, está más allá de los límites del arte" (pp. 62-63). A esto se refería Kant (2012) cuando señalaba que el arte es una "finalidad sin fin" (p. 287), una realidad autónoma cuyo

2 Helio Piñón (2006) distingue a las vanguardias artísticas que realizan una acción centrípeta sobre el propio arte y construyen -bajo un impulso estético autónomonuevas realidades formales (suprematismo, purismo y neoplasticismo) de las que ejercen una acción centrífuga y comunicativa (expresionismo, dadaísmo y surrealismo), supeditada a aspectos extra-artísticos.

3 Nueva traducción del original alemán Kritik der Urteilskraft (frente a la tradicional Crítica del Juicio) propuesta por Roberto R. Aramayo y Salvador Mas (Kant, 1790/2012). 
contenido se agota en su propio formarse. Esta obra de arte nueva -que no se remite a ninguna preexistencia- e independiente -tanto de la política o la religión como de la historia, la tecnología o la biología - interpela al observador para que adquiera un nuevo modo de conciencia a través de su mirada. Pero para ello es necesario que este cuente con una educación estética que le permita establecer juicios visuales.

A continuación, tras exponer sus principales enunciados, el formalismo se desarrolló a comienzos del siglo XX en Europa Central, mediante aportaciones sucesivas de autores destacados como Adolf von Hildebrand, Aloïs Riegl o Heinrich Wölfflin. ${ }^{4}$ Sus postulados tuvieron una gran repercusión sobre las artes plásticas, y alcanzaron a importantes autores como Paul Cezànne y a corrientes precursoras de la abstracción como el cubismo (Figuras 1 y 2). Durante la década de 1920, su influencia se dejó sentir sobre la arquitectura. Entonces apareció la arquitectura moderna: los arquitectos sintetizaron en su actividad a las corrientes críticas con el eclecticismo, y generaron una nueva manera constructiva de concebir en medio de una profunda crisis de la tradición figurativa y mimética del pasado.

A consecuencia de ello, aún hoy se mantiene la pugna entre dos metodologías antagónicas de diseño: La primera defiende la emancipación estética del arte figurativo, mientras que la segunda desconfía de los avances formalistas contenidos en la abstracción. Así las compara Carlos Martí (2000):

El procedimiento abstracto decanta el quehacer arquitectónico hacia la vertiente sintáctica, dando prioridad a las reglas de construcción formal del propio objeto; el interés se desplaza, entonces, desde los elementos hacia las relaciones que se establecen entre ellos y

4 A este respecto, Francisca Pérez Carreño (1999) apunta que: "De las ideas formalistas surgen una serie de métodos, de concepciones del arte y su historia, que tienen en común el tratar de delimitar criterios puramente artísticos. Las escuelas formalistas, por diversas que puedan ser sus interpretaciones, comparten este principio de autonomía[...] Su objeto de estudio es el desarrollo de la forma, considerada como lo específicamente artístico, en sus diferentes manifestaciones" (p. 256). hacia los principios de composición que las regulan. La elaboración figurativa otorga mayor peso a la vertiente semántica; adquiere, en este caso, una especial importancia la cuestión del carácter; por consiguiente, los códigos de significado que informan sobre el uso a que se destina el edificio, sobre su relevancia social o económica, o sobre los valores expresivos que la obra trata de manifestar, se convierten en el principal eje de acción del proyecto. (p. 8)

A pesar de todo, la arquitectura moderna (de origen formalista) tuvo que afrontar, desde el surgimiento de las vanguardias, dos dificultades. En primer lugar, el abuso de la composición abstracta elemental acarreó, con frecuencia, la implantación repetitiva de soluciones desarraigadas que apenas prestaron atención a su contexto. Al multiplicarse mediante la puesta en práctica de un planeamiento expeditivo, generaron barrios desangelados para poblaciones populares recientemente incorporadas a los suburbios urbanos. Pese a las declaratorias triunfales del funcionalismo, se pudo comprobar que muchas de estas ciudades no resultaron bien. En segundo lugar, los mismos arquitectos modernos que revolucionaron la estética occidental infundieron a la publicidad de sus actos la idea hegeliana del espíritu del tiempo o Zeitgeist. Mies van der Rohe (1982) insistía en que: "La arquitectura es la voluntad de una época traducida al espacio" (p. 25). Esta perspectiva condujo a ver sus obras como exponentes modélicos de acontecimientos ajenos al arte (innovaciones constructivas o arreglos funcionales), olvidando que estos sirvieron de pretexto para organizar la forma.

La incomprensión de la naturaleza formalista de la arquitectura indujo finalmente a la crítica posmoderna a rechazar por completo el international style, que se interpretó -dice Helio Piñón (2006) - como un estilo más basado en una figuratividad seca, asociada a un ascetismo próximo a una visión positivista de la ingeniería. Así se ha entendido la famosa metáfora de Le Corbusier que asegura que "una casa es una máquina de habitar" (1998, p. 73). No se suele advertir que esta frase no hace referencia a la mecanización de la arquitectura. Por el contrario, Charles Eduard 
Jeanneret ${ }^{5}$ reconoció -frente al abandono de los arquitectos figurativos- los atributos de la forma moderna en la obra de los ingenieros (Ozenfant \& Jeanneret, 2004).

En cualquier caso, las recusaciones posmodernas no han logrado imponer un marco estético superador. Su apuesta consiste más bien en negar dicha posibilidad, por desdén del método y rechazo de las teorías generales.

El arte contemporáneo, conceptual y discursivo, se distingue ahora por el fraccionamiento de sus propuestas, sujetas a interpretación. En este contexto, la forma ha dejado de ser la primera condición del arte y la arquitectura retrocede hasta asumir la lógica populista que requiere la mirada distraída que caracteriza al observador común, receptor pasivo de una avalancha incesante de imágenes sustraídas al juicio estético. ${ }^{6}$ El desinterés por la visualidad se compensa entonces sublimando la expresión que, aislada del rigor que proporciona la técnica, proyecta una subjetividad elaborada por la industria del entretenimiento. Así lo expone Theodor W. Adorno (2004):

Las personas embaucadas por la industria cultural y sedientas de sus mercancías se encuentran más acá del arte: por eso perciben su inadecuación al proceso vital de la sociedad de hoy (pero no la falsedad de este) con menos tapujos que quienes todavía recuerdan lo que en tiempos fue una obra de arte. Apremian a la desartificación del arte. (p. 30)

Mientras tanto, con el descuido de la visualidad se ha propagado en las escuelas de arquitectura el recurso al concepto: una herramienta creativa que surge como ensayo para evitar la banalidad de la figura, al adquirir el autor perspectiva del declive de la forma. Pero el abandono de la centralidad de la mirada ha alcanzado ya tal amplitud, que la aplicación del concepto se plasma en simples metáforas de inmediata representación icónica. De este modo, al emplear una idea o concepto, el diseñador contradice el carácter sensitivo

5 Conocido poco después como Le Corbusier.

6 Para Walter Benjamin (2003), "la arquitectura ha sido desde siempre el prototipo de una obra de arte cuya recepción tiene lugar en medio de la distracción" (p. 93). del arte, pues la comprobación racional de su presencia sustituye al juicio estético.

\section{Problemas en el aprendizaje del proyecto}

Es necesario rescatar al Diseño de sus problemas actuales, pero para conseguirlo se debe cuestionar una actitud que ha adquirido en los últimos tiempos un protagonismo inusitado: el afán de "innovar" sin descanso.

La necesidad de innovar nace de la mitología del progreso propagada por la Ilustración en el siglo XVIII. De la creencia de que la Humanidad transita por una vía de mejora permanente sin retroceso posible deriva la idea de que lo nuevo es necesariamente mejor que lo antiguo. La novedad se asocia entonces con un incremento de calidad. De lo nuevo se infiere aquello que es mejor a lo antiguo y su bondad se defiende contra toda evidencia. ${ }^{7}$ Es tan grande el afán por alcanzar la quimera de lo nuevo, que se disloca la noción misma de progreso. Se olvida incluso la relación que existe entre la apariencia fetichista de las cosas y la calidad resultante del proceso de su fabricación. La innovación mixtificada se transforma al fin en un mandato. Lo nuevo ya no es necesariamente mejor que lo antiguo, pero siempre se impone por presión de la moda.

En arquitectura, cada lustro aparecen nuevos postulados que pretenden suplantar por obsoletos a todos los anteriores. Ya no se parte de un bagaje recibido del pasado (denostado por caduco), sino de un vacío cultural desde el que se obtiene lo nuevo de la nada. La copia se considera inadecuada $y$, a menudo, se confunde con el plagio. Desde el principio, queda excluido de la docencia el análisis de

\footnotetext{
7 Este dogma ya fue criticado por Lewis Mumford (2002): "El hecho de que las ciudades del siglo XIII eran más alegres y más limpias y mejor ordenadas que las nuevas ciudades victorianas; el hecho que los hospitales fuesen más espaciosos y más higiénicos que sus sucesores victorianos; el hecho que en muchas partes de Europa el artesano medieval hubiese disfrutado ostensiblemente de un nivel de vida más alto que el esclavo paleotécnico, triunfalmente atado a una máquina semiautomática, estos hechos ni siquiera se les ocurrían a los exponentes del Progreso como posibilidades de investigación. Se descartaban automáticamente por la teoría misma" (p. 202).
} 
ejemplos, pues se teme que coarten la libertad personal. Para refundar la arquitectura a diario se ignoran en las aulas los ejemplos que podrían marcar un buen nivel de partida en la enseñanza y desarrollo del proyecto. Más bien, se abandona al proyectista a un estado de gracia con la esperanza de que consiga pautar en solitario su proceso de trabajo. Así se clausura el medio de proporcionar otros valores que, de acuerdo a unos principios consensuados, permitan afrontar con garantía el desempeño profesional.

Bajo esta óptica, se comprende que la creatividad se haya convertido en un valor crucial, que se cultiva, como acto mágico, para gestar productos nunca vistos. Con todo, la creatividad aplicada al arte tiene una trayectoria muy reciente, ya que se extendió en el siglo XIX y se popularizó en otros campos en el $\mathrm{XX}$. En sus inicios, era considerada un atributo divino. Tatarkiewicz (2015) comenta cómo era en el pasado:

La actitud de los antiguos hacia el arte puede expresarse de un modo más completo así: el arte no contiene ningún tipo de creatividad; es más, sería algo prejudicial que así fuese. La creatividad en el arte no sólo es imposible, sino indeseable, ya que el arte es una destreza, es decir, la destreza para fabricar ciertas cosas, y esta destreza presupone un conocimiento de las normas y la capacidad para aplicarlas: quien las conoce y sabe aplicarlas es un artista [...]El artista es un descubridor, no un inventor. (pp. 285-286)

Para los estudiantes de la Bauhaus, por ejemplo, la creatividad fue una importante facultad a desplegar en su trabajo. Johannes Itten, primer director (1919-1923) en Weimar del curso preliminar o Vorkurs, aseguraba (Wick, 1998) que su propósito era "liberar las fuerzas creadoras y con ello la capacidad artística de los estudiantes" (p. 88). Pero, en opinión de Itten, el aprendizaje del arte se desvirtúa al contactar con el diseño, pues está condicionado por otros requerimientos externos. Esta convicción lo llevó a enfrentarse con Walter Gropius, ${ }^{8}$ quien lo reemplazó en su cargo por László Moholy-Nagy, un autor más proclive a

8 Director de la Bauhaus entre 1919 y 1928. conciliar el arte con la técnica en beneficio de su integración social.

El individualismo creativo imposibilita el acercamiento de la arquitectura al conjunto de la sociedad, porque abandona el propósito de mejora colectiva que -a través del diseñoalentó al iniciarse el siglo XX en Alemania al Werkbund y a la Bauhaus. El proyecto se degrada al renunciar a la forma, que proporciona un orden visual. De este modo, la profesión queda relegada y renuncia a competir con ingenieros y constructores de mayor prestigio social. El proyecto de arquitectura se instituye en consecuencia oscilando entre dos extremos alienantes, operativos para el BAU: o bien se acoge a la repetición robotizada de estándares inmobiliarios u opta por insertarse en la ciudad como equipamiento de excepción. ${ }^{9}$

En el caso peruano, a menudo se soslaya que la precariedad es la norma que rige la construcción en gran parte del país. Este olvido tal vez se deba a la asunción de una falsa conciencia (la arquitectura kitsch) que, a la vez que deniega la realidad urbana, genera la desafección de los nuevos arquitectos ante la difícil tarea de mejorar los suburbios. ${ }^{10}$

\section{Metodología del taller}

¿Se puede trazar un método mejor para enseñar o aprender a diseñar arquitectura?

A pesar de las dificultades, es posible ensayar mejoras académicas. Uno de estos casos es la oportunidad que ahora se presenta en el Taller Básico 2 de la Facultad de Arquitectura y Urbanismo de la URP. Actualmente, este taller se encuentra en proceso de renovación y ha apostado por una pedagogía rigurosa para la enseñanza y el aprendizaje del diseño arquitectónico en los cursos iniciales de la carrera. Aquí se cuestiona la lectura ingenua de las nociones de innovación y creatividad.

BAU: Business as usual (negocios como siempre). Es una expresión atribuida a Winston Churchill.

10 Antonio Miranda (1999) destaca lo siguiente: "La arquitectura raras veces -y sólo en el mejor de los casosescapa de ser modelo ideológico del status. No en vano podemos llamarla televisión de la Antigüedad" (p. 184). 
En Perú, la carrera de Arquitectura se ordena en torno a cursos troncales de Diseño que en general se extienden por semestres, desde el acceso del alumno a la universidad (ciclo 1) hasta su salida (ciclo 10). En la Facultad de Arquitectura y Urbanismo de la URP, estos cursos se agrupan en talleres verticales. Un taller -integrado por varios docentes y numerosos estudiantes- es un área que se orienta a la enseñanza y aprendizaje mediante la puesta en práctica de ejercicios semejantes a los que acontecen en la realidad profesional. Un taller vertical es aquel que reúne en su seno asignaturas análogas en niveles consecutivos. En la URP, los Talleres Básicos proporcionan la formación inicial en los ciclos 1 y 2; después, los Talleres Integrales la desarrollan con extensión entre los ciclos 3 y 10. Tras superar el Taller Básico, el estudiante accede a un Taller Integral. La elección es libre y se puede cambiar de grupo al finalizar el ciclo académico.

Los talleres verticales tienen algunas ventajas: facilitan al estudiante la obtención de una experiencia recurrente en el trabajo de proyecto, con la coherencia que proporciona un método común para todos los cursos. En el caso del Taller Básico 2, esta recurrencia se produce en el análisis de los principios ordenadores de la forma y del espacio, y en la posterior aplicación de lo aprendido. Al mismo tiempo, este orden permite al estudiante aprender no solo de su interacción con los docentes, sino también de la relación que establece con alumnos de diversos niveles. Los talleres verticales pueden eludir la especialización horizontal en saberes específicos tangenciales al diseño y concentrarse en su principal cometido: la enseñanza constante y cíclica del proyecto de arquitectura.

El Taller Básico inicia al estudiante en su carrera y, a menudo, establece su primer encuentro consciente con la Arquitectura. Cristina Dreyfuss (2008) ha estudiado los primeros talleres de diseño en cuatro facultades de Lima y ha identificado en ellos diferentes enfoques:

a. El enfoque técnico-tectónico,

b. El espacio arquitectónico, c. La composición formal,

d. El usuario-comportamiento y función,

e. El ser humano-antropometría,

f. El entorno-el territorio.

Cabe destacar que uno de estos enfoques es la composición formal. La autora observa en su texto que en algunos talleres se trabaja con la noción de forma, pero no registra que esta idea se asocie en ellos a su interpretación estructural: "La mayoría de talleres, al mencionar sus preocupaciones centrales, se refieren tarde o temprano a la forma o al aspecto externo de la producción de los alumnos" (Dreyfuss, 2008, p. 25).

Por ello, el Taller Básico 2 ha decidido articular la fase inicial de una enseñanza análoga a la descrita por Helio Piñón (2005) en su libro El Proyecto como (Re)Construcción. Allí expone un método ensayado en la Escuela Técnica Superior de Arquitectura de Barcelona (ETSAB): la copia reproductiva y analógica de obras ejemplares. Con este método, el estudiante supera la simple imitación de figuras pintorescas, cuyo carácter a-sistémico entra en conflicto de modo invariable con los órdenes tectónico y programático que son propios del arte de construir.

En el curso de Diseño Arquitectónico 1 del Taller Básico 2 de la URP, se trabaja con dos categorías primordiales: el espacio y la forma. Sobre la trascendencia de la noción de espacio hay, en arquitectura, un cierto consenso. Se asume que la arquitectura es el arte del espacio. Como es bien sabido, fueron Aloïs Riegl y August Schmarsow (en De Fusco, 1976) quienes destacaron, a comienzos de siglo $\mathrm{XX}$, su importancia disciplinar. ${ }^{11}$ Sin embargo, no existe tal acuerdo cuando se habla de la forma. Por ello, se organiza su análisis en obras ejemplares. En el curso, se examinan los principios ordenadores de la forma -entendida como estructura- y se aplica el saber asimilado. A continuación, se estudia también

\footnotetext{
11 La idea de espacio en arquitectura fue expuesta, por primera vez, de modo manifiesto en El Arte Industrial Tardo-Romano (A. Riegl) de 1901 y en Los Principios Básicos de la Ciencia del Arte en la Transición de la Antigüedad a la Edad Media (A. Schmarsow) de 1905.
} 
el espacio arquitectónico y se descubren los elementos que lo conforman. Por último, se recrea lo aprendido con una propuesta libre. En el curso de Diseño Arquitectónico 2 se realizan las mismas tareas, pero se incluye la dimensión funcional de la arquitectura a un nivel básico.

Para que un estudiante consiga alcanzar sentido de la forma debe seguir un proceso de aprendizaje exhaustivo. En este proceso, el alumno transita -desde el análisis formal a la síntesis estructurada- dentro de una escala de complejidad ascendente:

a. Identificando los elementos plásticos que genera un sistema formal: El estudiante no debe concluir que reconoce lo que ve de manera inmediata. Es imprescindible que desglose con atención (con la ayuda del dibujo) los elementos plásticos fundamentales de la obra en estudio.

b. Reconociendo el sistema de relaciones que establecen estos mismos elementos: Tras el desglose previo de elementos plásticos, se plantean gráficamente las relaciones que establecen entre ellos. Estas relaciones son las que conducen a un sistema formal.

c. Generando otro sistema análogo al anterior: Realmente, el aprendizaje básico del diseño se inicia en sentido estricto, cuando el alumno -al adquirir la capacidad de replicar la forma- recrea formas análogas.

El aprendizaje del proyecto evoluciona de modo positivo desde el momento en que el alumno crea sistemas visuales nuevos, ordenados por él, y hace suya la tarea de generación de la forma. Para ello, practica en primer lugar la copia reproductiva. Luego, continúa con la copia analógica. Por último, realiza nuevas propuestas. Esta metodología está en las antípodas del plagio. Con ella, el estudiante vuelve a producir la obra y comprende en el camino sus principios ordenadores mediante un ejercicio exhaustivo de reconstrucción. Este trabajo confirma la autoría independiente y soslaya la imitación con la recreación. En los niveles superiores del aprendizaje, el proyecto de arquitectura se cumple cuando el estudiante es capaz de generar una estructura formal con identidad propia, y de adecuarla con éxito a un programa dado y a un sistema tectónico pertinente.

La finalidad no es imponer al alumnado referencias insuperables, tal y como entendió el clasicismo la arquitectura grecorromana. Es, más bien, a la inversa: el propósito es acercarlo a un modo libre, pero riguroso, de concebir. Esto no implica malinterpretar la circunstancia de que los pioneros modernos redujeran al mínimo la importancia de la historia en su trabajo. En su ruptura, necesitaron subrayar las diferencias adoptadas con un pasado decadente. En la actualidad, la fractura ya no divide el presente con el pasado, sino con el futuro: la arquitectura desaparece y primero se disipa en la universidad. Por este motivo, es imprescindible volver a fijar la mirada en la tradición. Así lo expresa Livio Vacchini (2007):

Los antiguos se hicieron amigos míos, hasta el punto de que llegué a creer que estaban vivos y que podía trabajar con ellos cotidianamente. La historia se me apareció entonces como una cadena de acontecimientos tendentes a la solución de ciertos problemas comunes $\mathrm{y}$, de repente, me sentí como un intermediario deseoso de ofrecer mi modesta contribución a la solución de estos problemas.

Hubo primero una fase de imitación. No hubiera osado contradecir a los maestros. Pero de pronto advertí que no pueden existir dos acciones idénticas, que cualquier acción que hagamos comporta un cierto grado de invención, y también me di cuenta de que nuestro trabajo nace de una operación crítica a partir de lo que ya existe y que la crítica más fértil es aquella en la que tenemos la valentía de cuestionar las obras maestras. (p. 7)

El trabajo de diseño podría volver a religarse con la tradición si se entiende que la forma subyace a la imagen contingente de la obra y que se transmite, en gran medida, a través del reconocimiento de arquetipos construidos: secuencias de eslabones formales que se suceden, actualizándose, a lo largo del tiempo.

\section{Conclusiones}

Como ya se ha expuesto, es importante que el estudiante de Arquitectura discrimine los 
conceptos de forma y de figura en la teoría del arte. Con frecuencia, estas ideas se confunden en la profesión con las de uso habitual en el idioma. La idea de forma -entendida como un sistema ordenado de elementos plásticos que se reconoce con la mirada- es una herramienta práctica de primera magnitud para el proyecto arquitectónico. Por el contrario, la figura es un estereotipo semántico que se mantiene inerte en su improbable coordinación con la tectónica de la obra. Para el formalismo, el significado es un rasgo que se ubica invariablemente, sea cual sea su naturaleza específica, fuera del ámbito artístico. Es la forma la que otorga identidad visual a la propuesta. Por ello, tanto si se está o no de acuerdo con la exclusión de la figura y su significado del arte de construir, se considera que conseguir sentido de la forma es un logro fundamental para el estudiante de diseño.

Los arquitectos posmodernos rechazan ( $\mathrm{u}$ olvidan) el manejo de la forma en su ejercicio habitual. En su lugar, confeccionan imágenes figurativas sin sopesar que el valor de la obra ya no viene dado por su dimensión visual, sino por su contenido narrativo. ${ }^{12}$ La exacerbación del fenómeno deriva al fin en la mitología de la creatividad y de la innovación, ajenas a la crítica. Pero la creatividad alejada de la experiencia diverge de la innovación

12 Como han reconocido de manera explícita los autores de Aprendiendo de Las Vegas, "Es posible que el solapado de disciplinas diluyera la arquitectura, pero en cambio enriqueció el significado" (Venturi, R., Scott Brown, D. \& Izenour, S., 2011, p. 27).

\section{Referencias}

Adorno, Th. W. (2004). Teoría estética. Madrid, España: Akal.

Agrupación Espacio. (1947). Expresión de principios. Recuperado de https://icaadocs.mfah.org/icaadocs/ELARCHIVO/ RegistroCompleto/tabid/99/doc/1126309/language/esMX/Default.aspx

Benjamin, W. (2003). La obra de arte en la era de su reproductibilidad técnica. México: Ítaca. auténtica. En su lugar, se retrocede hasta alcanzar formulaciones cada vez mas estrambóticas, semejantes a las que identificaron al peor eclecticismo del siglo XIX.

Muchas escuelas de Arquitectura asignan a la innovación un papel central en su enseñanza. Sin embargo, la novedad ilusoria oculta, en ciertas ocasiones, el desinterés por mejorar el muestrario de construcciones ordinarias (en el sentido material y visual) que -fruto de la especulación del suelo- puebla por todas partes ciudades y megápolis. El deseo de renovación permanente impide la estabilidad necesaria para organizar un medio urbano sostenible. Por ello, es necesario poner en marcha nuevas propuestas pedagógicas que reconduzcan la arquitectura a su centro de interés: la conjunción de los valores que la integran en un solo orden coherente o sistema tectónico, funcional y visual (firmitas, utilitas, venustas).$^{13}$ Esta es la tarea que ahora se emprende en el Taller Básico 2 de la URP: Frente a la novedad, se propone el análisis de la mejor arquitectura; frente a la creación, se plantea la recreación de ejemplos excelentes. De este modo, el estudiante educa su mirada, adquiere un bagaje estético genuino y elude la imitación vulgar basada en la apariencia.

$\mathrm{La}$ arquitectura se aprende siempre a partir de la arquitectura que ya existe.

13 Firmeza, utilidad, belleza. Legendaria tríada de Marco Lucio Vitruvio (1997), que aparece en el Libro I, Capítulo III, de su obra (p. 17).
Colquhoun, A. (2000). Forma y figura. En Cuaderno de Lectura Morfología. Cátedra de Javier García Cano (1) (pp. 88-100). Recuperado de http://www.catedragarciacano.com.ar/wp-content/uploads/2008/08/ cuaderno-de-lecturas-nro 1-parte-6.pdf

De Fusco, R. (1976). La idea de arquitectura: Historia de la crítica desde Viollet-le-Duc a Persico. Barcelona, España: Gustavo Gili. 
Dreyfuss, C. (2008). La Arquitectura en su enseñan$\mathrm{za}$ /aprendizaje en el primer Taller de Diseño. Investigaciones en Ciudad y Arquitectura, 1(1), 23-33. Recuperado de http://www.bibliotecacentral.uni.edu. pe/pdfs/CIUDADYARQUITECTURA/1,2008/ art_0002.pdf

Forma. (2014) En Diccionario de la Lengua Española (23 $3^{\mathrm{a}}$ ed.). Recuperado de http://dle.rae.es/srv/ fetch?id=IEvo $12 \mathrm{v}$

Figura. (2014) En Diccionario de la Lengua Española $\left(23^{\mathrm{a}}\right.$ ed.). Recuperado de http://dle.rae. es/?id=HsesZOK

Kant, I. (2012). Crítica del discernimiento (o de la facultad de juzgar). Madrid, España: Alianza Editorial.

Le Corbusier (1998). Hacia una arquitectura ( $2^{\mathrm{a}}$ ed.). Barcelona, España: Poseidón.

Martí Arís, C. (2000). Abstracción en arquitectura: Una definición. Documents Projectes Arquitectura, (16), 8.

Mies van der Rohe, L. (1982). Escritos, diálogos y discursos. Madrid, España: Colegio Oficial de Aparejadores y Arquitectos Técnicos de Madrid.

Miranda, A. (1999). Ni bufón ni robot: Manual para la crítica de arquitectura. Madrid, España: Cátedra.

Mumford, L. (2002). Técnica y civilización. Madrid, España: Alianza Editorial.
Ozenfant, A. \& Jeanneret, Ch. E. (2004). Acerca del purismo: Escritos 1918-1926 (2 ${ }^{\mathrm{a}}$ ed.). Madrid, España: El Croquis.

Pérez Carreño, F. (1999). El formalismo y el desarro$1 l o$ de la historia del arte. En V. Bozal (Ed.), Historia de las ideas estéticas y de las teorías artísticas contemporáneas (Vol. II, pp. 255-267). Madrid, España: Visor.

Piñón, H. (2006). Teoría del proyecto. Barcelona, España: Universitat Politècnica de Catalunya.

Piñón, H. (2005). El proyecto como (re)construcción. Barcelona, España: Universitat Politècnica de Catalunya.

Tatarkiewicz, W. (2015). Historia de seis ideas: Arte, belleza, forma, creatividad, mímesis, experiencia estética ( $8^{\mathrm{a}}$ ed.). Madrid, España: Tecnos.

Vacchini, L. (2007). Arquitectura, historia y rito. Documents Projectes Arquitectura, (23), 6-7.

Venturi, R., Scott Brown, D. \& Izenour, S. (2011). Aprendiendo de Las Vegas: El simbolismo olvidado de la forma arquitectónica. Barcelona, España: Gustavo Gili.

Vitruvio, M. L. (1997). Los diez libros de arquitectura. Barcelona, España: Iberia.

Wick, R. (1998). La pedagogía de la Bauhaus. Madrid, España: Alianza Editorial. 